\title{
Long non-coding RNA AB019562 promotes cell proliferation and metastasis in human hepatocellular carcinoma
}

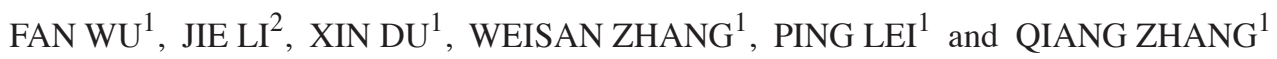 \\ ${ }^{1}$ Department of Geriatrics, Tianjin Medical University General Hospital, Tianjin Geriatrics Institute, \\ Tianjin 300052; ${ }^{2}$ Department of Cardiology, Tianjin Hospital, Tianjin 300202, P.R. China
}

Received March 6, 2016; Accepted February 15, 2017

DOI: $10.3892 / \mathrm{mmr} .2017 .6612$

\begin{abstract}
Increasing evidence has supported the prognostic and therapeutic values of long non-coding RNAs (LncRNAs) in human tumorigenesis. Hepatocellular carcinoma (HCC), as one of the most refractory diseases, continues to warrant investigation for novel clues to enable early diagnosis. In the present study, the role of LncRNA AB019562 in cell proliferation and metastasis was investigated in HCC. Reverse transcription-quantitative polymerase chain reaction analysis was performed to determine the expression of AB019562 in clinical HCC samples and cultured HCC cells. In addition, a specific small interfering RNA against AB019562 was designed and transfected into HCC cells. A3-(4,5dimethylthiazol-2-yl)-2,5-diphenyltetrazolium bromide assay and a transwell assay were used to assess the effects of AB019562 knockdown on cell proliferation and metastasis, respectively. The results revealed that the expression of AB019562 was increased 4-fold in the clinical HCC tissues, compared with adjacent non-cancerous tissue counterparts. AB019562 was differentially expressed in the HCC cell lines. The knockdown of AB019562 reduced the rate of cell proliferation by $28.6 \%$ in HepG2 cells and by $24 \%$ in SMMC-7721 cells. Cell cycle assays revealed that the proportion of cells in the $\mathrm{G} 0 / \mathrm{G} 1$ phase was significantly increased, whereas those in the $\mathrm{S}$ and $\mathrm{G} 2 / \mathrm{M}$ phases were decreased in the AB019562-knockdowncells. The results of the transwell assay showed that the knockdown of AB019562 inhibited cell migration abilities by up to $67 \%$ in the HepG2 cells and 63\% in the SMMC-7721 cells, and significantly suppressed invasive abilities by up to $75 \%$ in the HepG2 cells and 60\% in the SMMC-7721 cells. Furthermore, AB019562 knockdown increased the apoptotic rates of the two cell lines and activated the expression of caspase-3, but not caspase-8. These data demonstrated the pro-oncogenic
\end{abstract}

Correspondence to: Dr Qiang Zhang, Department of Geriatrics, Tianjin Medical University General Hospital, Tianjin Geriatrics Institute, 154 Anshan Road, Tianjin 300052, P.R. China

E-mail: qiangzhang8877@sina.com

Key words: long non-coding RNA, AB019562, proliferation, metastasis, apoptosis, hepatocellular carcinoma properties of $\mathrm{AB} 019562$ and suggested that $\mathrm{AB} 019562$ may serve as a novel biomarker for the diagnosis and treatment of patients with HCC.

\section{Introduction}

Hepatocellular carcinoma (HCC) is the fifth most prevalent malignancy worldwide and the second most common cause of cancer-associated mortality in eastern and western countries $(1,2)$. Each year, $>700,000$ patientcare diagnosed with $\mathrm{HCC}$ and morbidity rates of HCC continue to increase, possibly due to deterioration of environment, unhealthy dietary habits and other associated factors (3). The traditional therapeutic strategy for HCC is surgical resection, however, this is only able to cure patients at an early stage of the disease (4). For patients in the late stage, the introduction of sorafenib can improve survival rates, but remains limited (5). Despite substantial progression in therapeutics in previous decades, the prevention and treatment of HCC remains challenging. Thus, the identification of novel molecular markers to diagnose HCC at an early stage is of high priority.

Long non-coding RNAs (LncRNAs) are well-defined as a class of RNAs with a length of $>200$ nucleotides and $<100,000$ nucleotides, which are unable to translate proteins (6). LncRNAs can be classified into five categories by their locations at nearby genes: Sense, antisense, bidirectional, intronic and intergenic (7). LncRNAs have been demonstrated to be involved in various biological processes, including chromatin modification, transcription, translation, posttranscriptional processing and posttranslational processing $(8,9)$. Of note, studies have shown that certain LncRNAs can function as oncogenes or tumor suppressors, and that aberrant expression of LncRNAs may contribute to tumorigenesis $(10,11)$.

Emerging evidence has also linked LncRNAs with multiple physiological and pathological processes of HCC, including cell proliferation, metastasis, apoptosis and metabolism (12). For example, LncRNA AFAP1-AS1 functions to promote cell proliferation and metastasis, and upregulates the RhoA/Rac2 signing pathway in HCC (13). LncRNA DILC has been shown to repress the self-renewal of liver cancer stem cells by inhibiting the autocrine interleukin-6/signal transducer and activator of transcription 3 axis (14). Thus, LncRNAs have been recognized as potential markers in the progression of HCC. 
In the present study, the role of LncRNA AB019562 in cell proliferation and metastasis was examined in HCC. A specific small interfering (si)RNA against AB019562 was used to decrease the expression of AB019562 in HCC cell lines. The effects of AB019562 depletion on cell proliferation, metastasis and apoptosis were examined using a 3-(4,5-dimethylthiazol-2-yl)-2,5-diphenyltetrazolium bromide (MTT) assay, transwell assay and apoptosis detection kit, respectively. The results indicated the pro-oncogenic property of AB019562 in HCC and may provide novel clues for the early clinical diagnosis and treatment of HCC.

\section{Materials and methods}

Human samples. Clinical HCC tissue samples and samples of the adjacent non-cancerous tissues were obtained from 50 patients who had undergone surgical resection of $\mathrm{HCC}$ in the Department of Geriatrics, Tianjin Medical University General Hospital (Tianjin, China) between July 2012 and June 2015, in a non-identifiable manner and without additional clinical information. Prior to surgery, no chemotherapy or radiotherapy treatment was administered to the patients. All patients confirmed their full intention to be involved in the study and written informed consent was obtained. The present study was approved by the Research Ethics Committee of Tianjin Medical University General Hospital. All collected samples were immediately frozen in liquid nitrogen and stored at $-70^{\circ} \mathrm{C}$ until RNA isolation.

Cell culture. The SMMC-7721, PLC/PRF/5 and C3AHCC cell lines, and the normal THLE-3 liver cell line were all commercially obtained from American Type Culture Collection (Manassas, VA, USA). The HepG2 HCC cell line was purchased from Shanghai Cell Bank of the Chinese Academy of Sciences (Shanghai, China). All cells were cultured in Eagle's minimum essential medium (EMEM; American Type Culture Collection) supplemented with $10 \%$ fetal bovine serum (FBS; Gibco; Thermo Fisher Scientific, Inc., Waltham, MA, USA). During the experiments, the cells were maintained in an incubator containing $5 \% \mathrm{CO}_{2}$ at $37^{\circ} \mathrm{C}$.

Reverse transcription-quantitative polymerase chain reaction (RT-qPCR) analysis. RNAs from the human samples and cultured cells were extracted using TRIzol reagent (Takara Biotechnology, Co., Ltd., Dalian, China) according to the manufacturer's protocol. The isolated RNAs were eluted with ribonuclease-free water and quantified using a Nanodrop 2000 spectrophotometer (Invitrogen; Thermo Fisher Scientific, Inc.) by detecting the optical density (OD) ${ }_{260}$ and $\mathrm{OD}_{280}$. Complementary DNA (cDNA) was reverse transcribed from a total of 1ug RNAs using PrimeScript Reverse Transcriptase (Takara Biotechnology, Co., Ltd.) with a volume of $20 \mu \mathrm{l}$, as per the manufacturer's instructions. qPCR was performed using SYBR Green reagent (Takara Biotechnology, Inc.) in an ABI 7900 machine (Applied Biosystems, Foster City, CA, USA) and a total of $1 \mu \mathrm{l}$ cDNA was added into each well. The thermocycling conditions were as follows: $95^{\circ} \mathrm{C}$ for $5 \mathrm{~min}$, followed by 40 cycles of $95^{\circ} \mathrm{C}$ for $15 \mathrm{sec}$ and $60^{\circ} \mathrm{C}$ for $30 \mathrm{sec}$. The following primers were used in the reaction, as described previously (15): AB019562, forward 5'-GGATGTCAGGTC
TGCGAAACT-3' and reverse 5'-GATAGTGTGGTTTAT GGACTGAGGT-3'; GAPDH, forward 5'-GGGAAACTG TGGCGTGAT-3' and reverse 5'-GAGTGGGTGTCGCTG TTGA-3'. The RT-qPCR analysis was performed as previously described (15).

siRNA interference. The specific siRNA against LncRNA AB019562 (siAB019562) was designed and synthesized by GenePharm Co., Ltd. (Shanghai, China). The siRNAs were dissolved in $\mathrm{ddH}_{2} \mathrm{O}$ at a concentration of $20 \mu \mathrm{M}$. The negative control siRNA and the specific siAB019562 were transfected into cells using Lipofectamine 2000 (Invitrogen; Thermo Fisher Scientific, Inc.) according to the manufacturer's protocol. Briefly, the cells were washed with pre-warmed PBS and replaced with serum-free medium. The mixture of Lipofectamine 2000 and siRNAs was incubated for $20 \mathrm{~min}$ and then added to each well containing cells at a confluence of $60 \%$. Cells were incubated at $37^{\circ} \mathrm{C}$ for $72 \mathrm{~h}$ post-transfection, and then used for subsequent analyses.

Cell viability assay. An MTT assay (Promega Corporation, Madison, WI, USA) was used to measure cell proliferative abilities, according to the manufacturer's protocol. Briefly, theHepG2 and SMMC-7721 cells, at an initial concentration of $2 \times 10^{3} /$ well, were seeded into 96 -well plates in EMEM supplemented with $10 \% \mathrm{FBS}$ in a $37^{\circ} \mathrm{C}$ humidified incubator with $5 \% \mathrm{CO}_{2}$. Each experimental group of cells was divided into six wells and the cultural medium was refreshed every other day. At each time point (days 1, 2, 3,4 and 5 post-transfection), the cell numbers were determined by detecting the absorbance at $450 \mathrm{~nm}$ on a Tecan microplate reader (Tecan Group, Ltd., Männedorf, Switzerland). Proliferation rate was plotted as a ratio relative to day 1 .

Cell cycle analysis. The cells were seeded into 96-well plates and incubated for $24 \mathrm{~h}$, following which the siRNAs were transfected into the corresponding group. At $72 \mathrm{~h}$ post-treatment, the HepG2 and SMMC-7721 cells were harvested and co-incubated with pre-cooled ethanol overnight. On the subsequent day, the cells $\left(3 \times 10^{4}\right.$ cells $)$ were treated with $50 \mu \mathrm{g} / \mathrm{ml}$ of RNase in a $37^{\circ} \mathrm{C}$ incubator for $1 \mathrm{~h}$, following which $20 \mu \mathrm{g} / \mathrm{ml}$ of propidium iodide (PI) was added to the cells. The mixture was maintained at $4^{\circ} \mathrm{C}$ for an additional $30 \mathrm{~min}$ in the absence of light. The DNA content was detected using flow cytometry.

Transwell assay. The HepG2 and SMMC-7721 cells were cultured in 24-well plates and transfected with siRNAs. At $48 \mathrm{~h}$ post-transfection, the cells were harvested with serum-free EMEM, following which $150 \mu$ l of cell suspension $\left(3 \times 10^{4}\right.$ cells) was added to the upper chamber (Corning Incorporated, New York, NY, USA), and the lower chamber was filled with $600 \mu \mathrm{l}$ culture medium containing 10\% FBS. For the invasion assay, the membrane was pre-coated with Matrigel (Corning Incorporated) for $6 \mathrm{~h}$ at $37^{\circ} \mathrm{C}$ prior to the experiments. Following incubation for $12 \mathrm{~h}$ at $37^{\circ} \mathrm{C}$, the cells in each group were fixed with ice-cold methanol for $10 \mathrm{~min}$ and stained with crystal violet for $5 \mathrm{~min}$. Images were captured under an inverted light microscope (TS100; Nikon Corporation, Tokyo, Japan). 

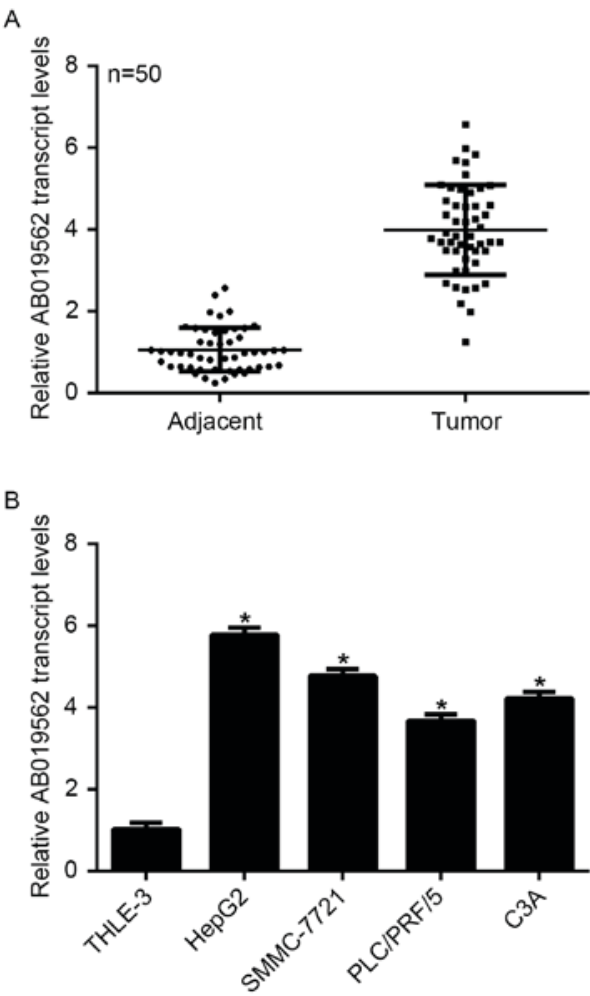

Figure 1. LncRNA AB019652 is upregulated in HCC tissues and cultured HCC cells. (A) Tissue samples from 50patients with HCC were collected and RT-qPCR analysis was performed to examine the expression of LncRNA AB019562 in the HCC tissues and paired adjacent non-cancerous tissues. (B) Four HCC cell lines and a normal liver cell line were examined using RT-qPCR analysis. The expression of AB019562 was significantly upregulated in HCC cells, compared with normal liver (THLE-3) cells. " $\mathrm{P}<0.05$ vs. THLE-3 cells. HCC, hepatocellular carcinoma; LnRNA, long non-coding RNA; RT-qPCR, reverse transcription-quantitative polymerase chain reaction.

Detection of apoptosis. Flow cytometry was used to determine the apoptotic rates of cells following siAB019562 treatment. Briefly, the HepG2 and SMMC-7721 cells were harvested with low-speed centrifugation $\left(850 \mathrm{~g}, 4^{\circ} \mathrm{C}, 5 \mathrm{~min}\right)$ and washed three times with PBS. The cells were then suspended in $100 \mu \mathrm{l}$ staining buffer with $5 \mu \mathrm{l}$ of Annexin V-APC $(20 \mu \mathrm{g} / \mathrm{ml})$ and $5 \mu \mathrm{l}$ PI (50 $\mu \mathrm{g} / \mathrm{ml}$; BD Pharmingen, San Diego, CA, USA). The cells found to be Annexin V-APC-positive and PI-negative were identified as early apoptotic cells, and those positive for Annexin V-APC and PI were identified as late apoptotic cells. Each experiment was repeated at least 3 times in triplicate.

Determination of relative caspase activity. The activities of caspase- 3 and caspase- 8 were determined using a Caspase-3 activity kit and a Caspase- 8 activity kit, respectively, according to the manufacturer's protocol (Beyotime Institute of Biotechnology, Nantong, China). Briefly, following siRNA transfection, $2 \times 10^{6}$ cells in 6 -well plates were lysed using lysis buffer (as provided in the kit) at $4^{\circ} \mathrm{C}$ for $15 \mathrm{~min}$, centrifuged at $600 \times \mathrm{g}$ at $4^{\circ} \mathrm{C}$ for $15 \mathrm{~min}$, and the resulting cell lysates were analyzed for protein concentration by bicinchoninic assay (Beyotime Institute of Biotechnology). An aliquot of $10 \mu \mathrm{l}$ proteins from the cell lysates were added to 96-well plates and mixed with $80 \mu \mathrm{l}$ reaction buffer containing caspase substrate (2 mM). Following incubation for $4 \mathrm{~h}$ at $37^{\circ} \mathrm{C}$, caspase
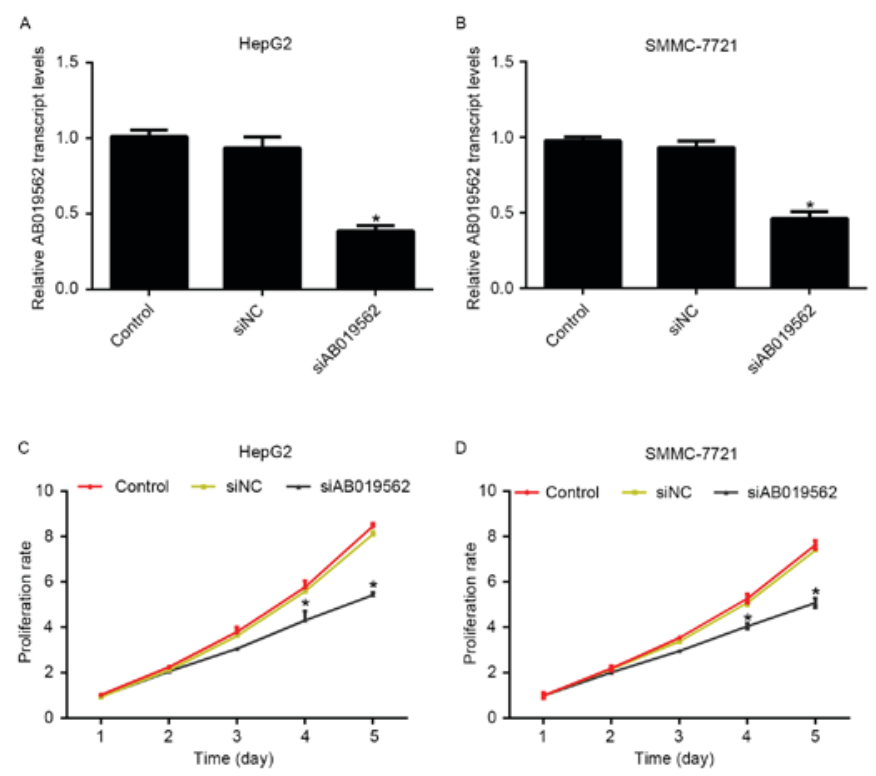

Figure 2. Knockdown of AB019562 inhibits proliferation of hepatocellular carcinoma cells. (A) Relative transcript levels of AB019562 in HepG2 cells transfected with specific siRNA against AB019562 (siAB019562). (B) Relative transcription levels of AB019562 in SMMC-7721 cells transfected with siAB019562. (C) Effects of AB019562 knockdown on cell proliferation were assessed over a 5-day period. In HepG2 cells, the proliferation rate was significantly decreased, whereas the level of AB019562 was reduced. (D) Proliferation rate of SMMC-7721 cells was significantly decreased, particularly on days 4 and 5 post-siAB019562 treatment. ${ }^{*} \mathrm{P}<0.05$ vs. control.si/siRNA, small interfering RNA; NC, negative control.
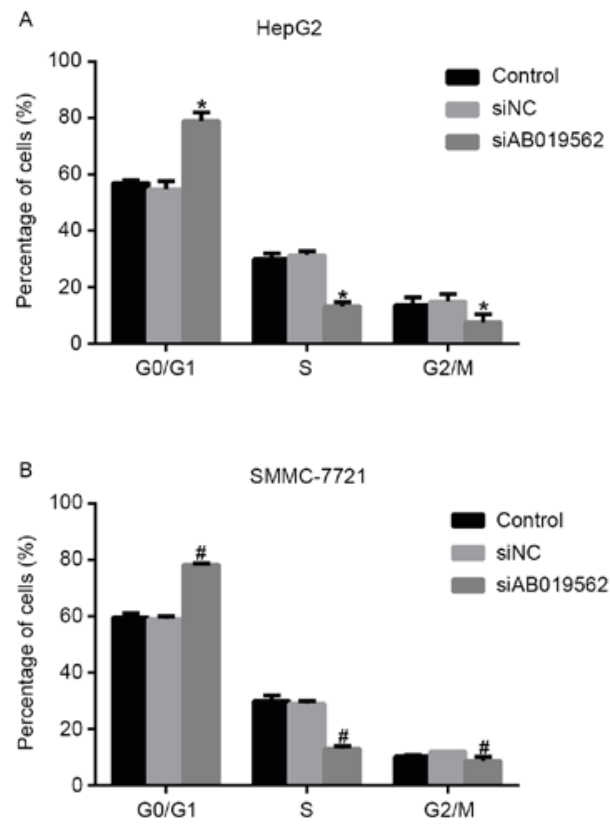

Figure 3. Knockdown of AB019562 arrests cell cycle at the G0/G1 phase in hepatocellular carcinoma cells. (A) Percentages of siAB019562-treated HepG2 cells in the G0/G1, S and G2/M phases were determined using flow cytometry. "P<0.05 vs. control. (B) Knockdown of AB019562 increased the percentage of SMMC-7721 cells at the G0/G1 phase, but decreased the percentages of cells at the $\mathrm{S}$ and $\mathrm{G} 2 / \mathrm{M}$ phases. ${ }^{\#} \mathrm{P}<0.05$ vs. control. si, small interfering RNA; NC, negative control.

activities were determined using a Tecan microplate reader at an absorbance of $405 \mathrm{~nm}$. 

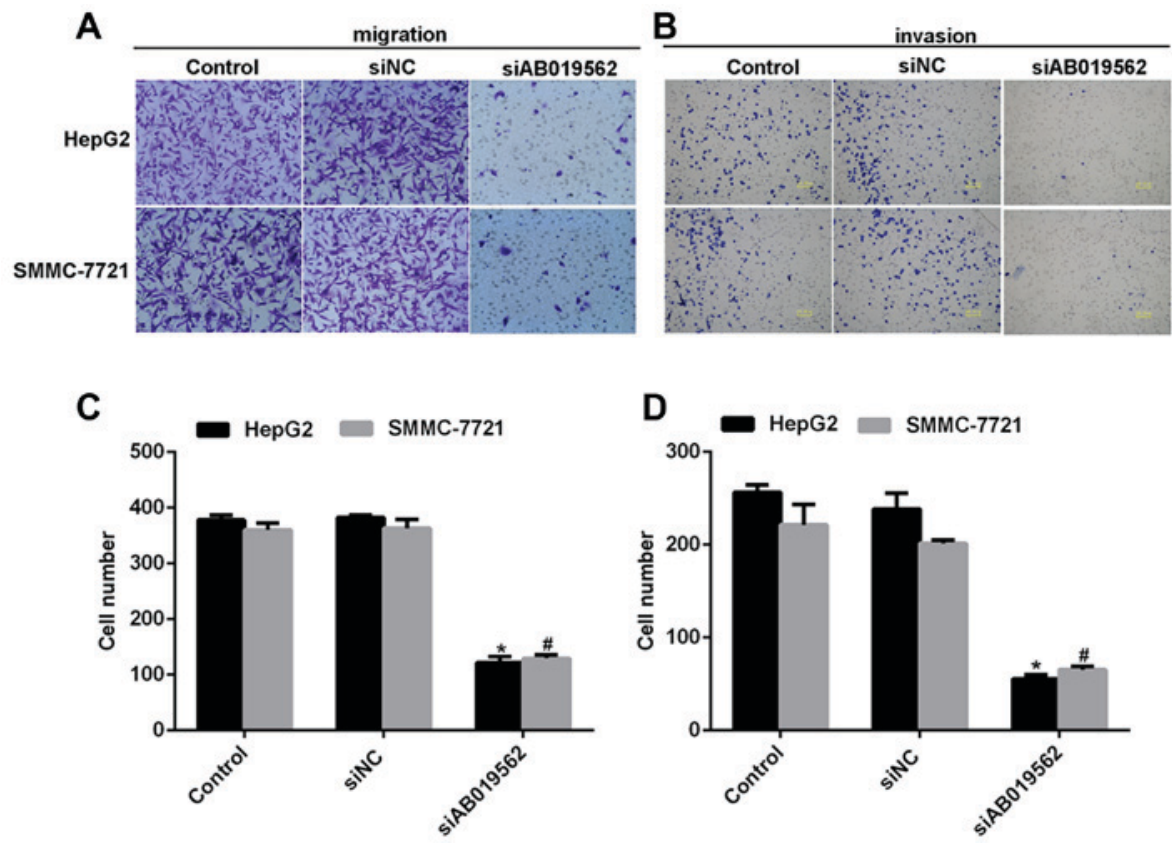

Figure 4. Knockdown of AB019562 suppresses the metastasis of hepatocellular carcinoma cells. (A) Representative images of cell migration assays off HepG2 and SMMC-7721 following transfection with the specific siRNA against AB019562 (siAB019562). Original magnification, x200. (B) Representative images of cell invasion assays of HepG2 and SMMC-7721 cells treated with siAB019562. Original magnification, x200. (C) Numbers of transmigrated cells in five fields of each image were counted. Quantification of the migrated HepG2 and SMMC-7721 cells. (D) Quantification of the invaded HepG2 and SMMC-7721 cells. "P<0.05 vs. control in HepG2 cells; ${ }^{\#} \mathrm{P}<0.05$ vs. control in SMMC-7721 cells. si/siRNA, small interfering RNA; NC, negative control.

Statistical analysis. All data are presented as the mean \pm standard deviation. Each experiment was repeated at least 3 times in triplicate unless otherwise stated. Statistical differences between two groups were evaluated by Student's t-test using GraphPad Prism version 5.0 (GraphPad Software, Inc., La Jolla, CA, USA). P<0.05 was considered to indicate a statistically significant difference.

\section{Results}

LncRNA AB019652 is upregulated in HCC tissues and cultured HCC cells. To examine the expression of LncRNA AB019562 in HCC, tissue samples from 50 patients with $\mathrm{HCC}$, including cancerous tissues and adjacent non-cancerous tissues, were obtained. As shown in Fig. 1A, the transcription level of AB019562 was 4-fold higher in the HCC samples, compared with that in the adjacent the non-cancerous samples $(\mathrm{P}<0.05)$. The four HCC cell lines and a normal liver cell line were examined to determine the transcription ofAB019562 in vitro. The results showed that, compared with the THLE-3 normal liver cells, HepG2 cells exhibited the highest level of AB019562 (6-fold increase), followed by the SMMC-7721 cells (5-fold increase). The transcription of AB019562 was also significantly increased in the PLC/PRF/5 and C3A cells (Fig. 1B). The HepG2 and SMMC-7721 cells were selected for subsequent analyses. These data suggested that AB019562 was significantly overexpressed in HCC in vivo and in vitro.

Knockdown of AB019562 inhibits cell proliferation in HCC cells. To evaluate the role of AB019562 in HCC cell proliferation, specific siRNA against AB019562 (siAB019562) was designed and transfected into the HepG2 and SMMC-7721 cells. The transcription levels of AB019562 were decreased by $58 \%$ in HepG2 cells (Fig. 2A) and 51\% in SMMC-7721 cells (Fig. 2B) following transfection with siAB019562, which suggested the high efficiency of the synthesized siRNA. With this effective siRNA, cell proliferative rates were examined following siRNA transfection using MTT assays. For the first 3 days post-siRNA treatment, no significant changes in the viability of the two cell lines were observed, compared with the control cells. However, on day 4 post-AB019562 knockdown, the proliferation rates were decreased by $28.6 \%$ in the HepG 2 cells and 24\% in the SMMC-7721. This inhibitory effect was more pronounced on day 5 following AB019562 knockdown in the HCC cells (Fig. 2C and D). These results suggested that AB019562 promoted cell proliferation in the HCC cells in vitro.

Knockdown of AB019562 arrests cell cycle at the G0/G1 phase in HCC cells. The present study subsequently analyzed cell cycle progression. As shown in Fig. 3A, when AB019562 was knocked down in the HepG2 cells, the proportion of cells in the $\mathrm{G} 0 / \mathrm{G} 1$ phase increased by $22 \%$, whereas the proportions of cells in the $\mathrm{S}$ phase and G2/M phase decreased by 14 and $8 \%$, respectively. Similarly, in the siAB019562-transfected SMMC-7721 cells, the percentage of cells in the G0/G1 phase was elevated by $18 \%$, whereas the percentages of cells in the $\mathrm{S}$ phase and $\mathrm{G} 2 / \mathrm{M}$ phase decreased 12 and $9 \%$, respectively (Fig. 3B). These data suggested that the knockdown of LncRNA AB019562 arrested cell cycle in the G0/G1 phase in HCC cell lines. The cell cycle arrest following AB019562 knockdown provided further support that AB019562 promoted the proliferation of HCC cells.

Knockdown of AB019562 suppresses the metastasis of HCC cells. Subsequently, a transwell assay was performed to 

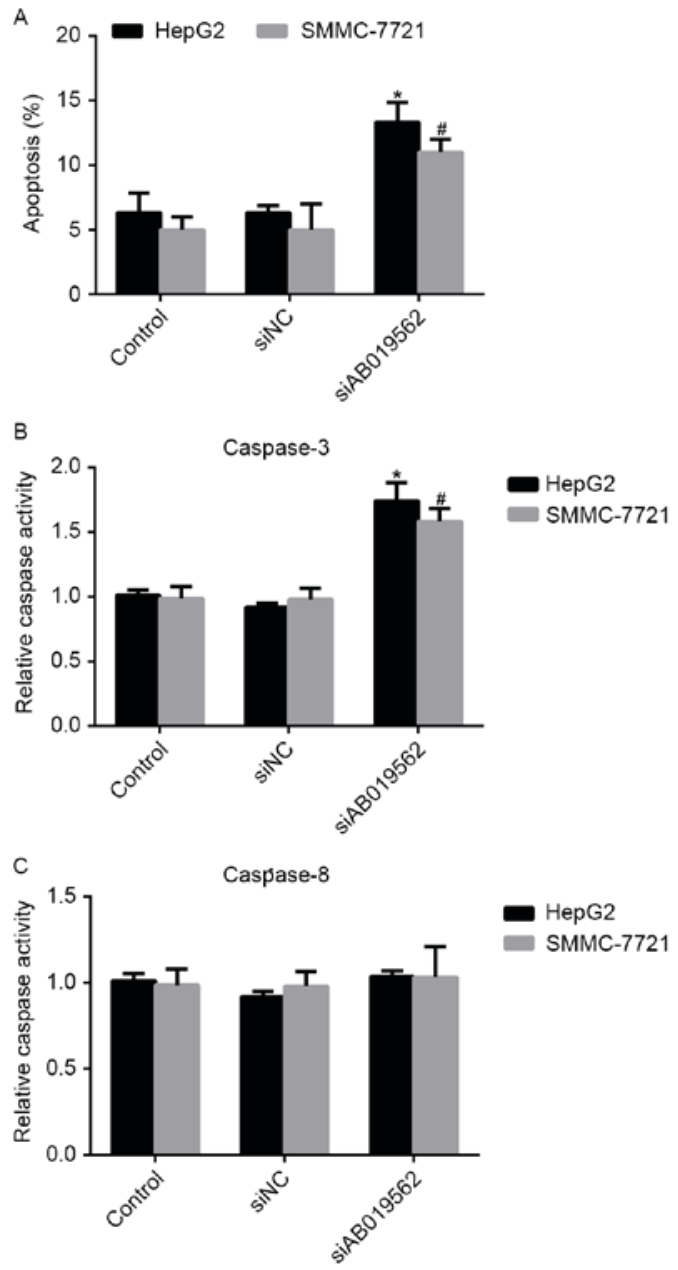

Figure 5. Knockdown of AB019562 induces apoptosis and affects the activity of caspase-3 in hepatocellular carcinoma. (A) Cell apoptosis was increased when HepG2 and SMMC-7721 cells were transfected with siAB019562. (B) Relative activities of caspase-3 were evaluated upon siAB019562 treatment in the two cell lines. (C) Relative activities of caspase- 8 remained unchanged, even following siAB019562 transfection. ${ }^{*} \mathrm{P}<0.05$ vs. control in HepG2 cells; ${ }^{*} \mathrm{P}<0.05$ vs. control in SMMC-7721 cells. si, small interfering RNA; NC, negative control.

examine the effects of AB019562 knockdown on cell migration (Fig. 4A) and invasive abilities (Fig. 4B) in the HCC cell lines. The results showed that transmigrated cells were visually decreased when the cells were transfected with siAB019562 (Fig. 4A and B). Quantification of the transmigrated cells further supported that migration abilities were reduced by siAB019562 transfection, with decreases by up to $67 \%$ in HepG2 cells and 63\% in SMMC-7721 cells (Fig. 4C). Similarly, it was also shown that $75 \%$ of HepG 2 cells and $60 \%$ of SMMC-7721 cells were inhibited from invading through the Matrigel upon siAB019562 treatment in the invasion assay (Fig. 4D). These data suggested that AB019562 promoted cell migration and invasion in the HCC cells in vitro.

Knockdown of AB019562 induces cell apoptosis and affects the activity of caspase-3 in HCC. Cell apoptosis has been demonstrated to be important in the tumorigenesis of HCC (16). Therefore, the present study examined the effects of AB019562 knockdown on the apoptosis of HCC cells. It was shown that, compared with the control cells, the percentages of apoptotic HepG2 and SMMC-7721 cells were increased by 8 and 7\%, respectively, following AB019562 knockdown (Fig. 5A). The activities of caspase- 3 and caspase- 8 in the HCC cells were also examined. As shown in Fig. 5B, AB019562 knockdown increased the activity of caspase- 3 by $60 \%$ in the HepG2 cells and 50\% in the SMMC-7721 cells. However, the activity of caspase- 8 remained unchanged, even when cells were transfected with siAB019562 (Fig. 5C). Taken together, these results confirmed that the knockdown of AB019562 induced cell apoptosis and promoted the activity of caspase-3, but not caspase-8.

\section{Discussion}

HCC is frequently one of the top five causes of liver cirrhosis. However, the early diagnosis of HCC remains challenging $(17,18)$. The primary treatment for HCC is surgery, which accounts for a five-year survival rate of $30-70 \%(19,20)$. However, $<20 \%$ of patients with HCC are eligible for surgery following confirmation of diagnosis (21), indicating the necessity of diagnosing $\mathrm{HCC}$ at an early stage. The most refractory limitation in the early detection and prevention of HCC is the absence of symptoms (22). Therefore, an increasing number of studies have focused on identifying novel biomarkers, which can be used to diagnose and prevent $\mathrm{HCC}$ in the early stage $(23,24)$.

LncRNAs are distinct from housekeeping RNAs, including tRNAs, rRNAs and siRNAs (25). They are important in various aspects of cell biology. The aberrant expression of LncRNAs is a basic feature of several types of cancer, and has been shown to affect cell proliferation, metastasis and apoptosis in human cancer $(26,27)$. The LncRNA AB019562 was first identified by Zhou et al using gene microarray analysis (28). In this pioneer study, AB019562 was shown to be upregulated in human hypopharyngeal squamous cell carcinoma. However, the role of AB019562 in HCC and the detailed mechanisms underlying how AB019562 regulates the tumorigenesis of $\mathrm{HCC}$ remain to be fully elucidated.

In the present study, the transcription levels of AB019562 were determined in HCC tissues and in a series of $\mathrm{HCC}$ cell lines. It was shown that the expression of AB019562 was markedly upregulated in HCC. Furthermore, it was observed that the knockdown of AB019562 significantly reduced the rate of cell proliferation and arrested cell cycle at the G0/G1 phase, suggesting the promotion of proliferation by AB019562. The induction of cell apoptosis by AB019562 knockdown further confirmed that AB019562 functioned to promote cell proliferation in HCC, as the induction of apoptosis is a sound basis for the inhibition of proliferation (16). In addition, the knockdown of AB019562 impaired cell migration and invasion abilities in the HCC cell lines. These data demonstrated that AB019562 promoted cell proliferation and metastasis in HCC.

However, whether the intrinsic or extrinsic apoptotic signal pathway predominantly contributes to the AB019562-mediated biological changes remains to be elucidated. The induction of apoptosis usually has two signaling pathways, the intrinsic and extrinsic pathways (29). The initiation of the intrinsic pathway is associated with the pro-apoptotic factors, B-cell lymphoma 2 (Bcl-2)-associated X protein and Bcl-2-associated death promoter, which leads to increased permeability of the 
mitochondria membrane, loss of membrane potential and the release of cytochrome $C$ into the cytosol. The intrinsic pathway is associated with activated caspase-3, whereas the extrinsic pathway is associated with the activation of caspase-8 (30). As shown in Fig. 5C, the activities of caspase- 8 were stable upon siAB019562 administration, which indicated that the extrinsic pathway may not be critically involved. Instead, the relative activities of caspase-3 were markedly increased following AB019562 knockdown in HepG2 and SMMC-7721 cells. This observation indicated that the intrinsic pathway maybe involved in the induction of apoptosis by siAB019562 transfection. However, further investigations are required to systemically reveal the detailed mechanisms.

In conclusion, the present study examined the role of LncRNA AB019562 in human HCC in vivo and in vitro. AB019562 was expressed at high levels in patients with HCC and cultured HCC cells. The knockdown of AB019562 caused cell cycle arrest at the G0/G1 phase, leading to eventual cell apoptosis and thereby inhibiting the proliferation of $\mathrm{HCC}$ cells. Furthermore, the knockdown of AB019562 impaired cell migration and invasion of the HepG2 and SMMC-7721 cells. These data suggested that AB019562 may promote cell proliferation and metastasis in $\mathrm{HCC}$, and provided evidence that AB019562 may serve as a novel biomarker and therapeutic target for the diagnosis and clinical treatment of HCC.

\section{Acknowledgements}

This study was sponsored by National Natural Science Foundation of China (grant nos. 81670086 and 81370183), Tianjin Natural Science Foundation (grant no. 14JCYBJC27800) and International S\&T Cooperation Program of China (grant no. 2015DFA50310).

\section{References}

1. Laursen L: A preventable cancer. Nature 516 (Suppl): S2-S3, 2014.

2. Park YN: Update on precursor and early lesions of hepatocellular carcinomas. Arch Pathol Lab Med 135: 704-715, 2011.

3. Maluccio M and Covey A: Recent progress in understanding, diagnosing, and treating hepatocellular carcinoma. CA Cancer J Clin 62: 394-399, 2012.

4. Forner A, Llovet JM and Bruix J: Hepatocellular carcinoma. Lancet 379: 1245-1255, 2012.

5. Worns MA and Galle PR: HCC therapies-lessons learned. Nat Rev Gastroenterol Hepatol 11: 447-452, 2014.

6. Guttman M and Rinn JL: Modular regulatory principles of large non-coding RNAs. Nature 482: 339-346, 2012.

7. Tang JW, Jiang R, Deng L, Zhang X, Wang K and Sun B: Circulation long non-coding RNAs act as biomarkers for predicting tumorigenesis and metastasis in hepatocellular carcinoma. Oncotarget 6: 4505-4515, 2015

8. Huarte M, Guttman M, Feldser D, Garber M, Koziol MJ, Kenzelmann-Broz D, Khalil AM, Zuk O, Amit I, Rabani M, et al: A large intergenic noncoding RNA induced by p53 mediates global gene repression in the p53 response. Cell 142: 409-419, 2010.

9. Bussemakers MJ, van Bokhoven A, Verhaegh GW, Smit FP, Karthaus HF, Schalken JA, Debruyne FM, Ru N and Isaacs WB: DD3: A new prostate-specific gene, highly overexpressed in prostate cancer. Cancer Res 59: 5975-5979, 1999.

10. Guo XQ, Xia J and Deng K: Long non-coding RNAs: Emerging players in gastric cancer. Tumor Biol 35: 10591-10600, 2014.
11. He Y, Meng XM, Huang C, Wu BM, Zhang L, Lv XW and Li J: Long noncoding RNAs: Novel insights into hepatocelluar carcinoma. Cancer Lett 344: 20-27, 2014.

12. Ulitsky I and Bartel DP: lincRNAs: Genomics, evolution, and mechanisms. Cell 154: 26-46, 2013.

13. Zhang JY, Weng MZ, Song FB, Xu YG, Liu Q, Wu JY, Qin J, Jin T and Xu JM: Long noncoding RNA AFAP1-AS1 indicates a poor prognosis of hepatocellular carcinoma and promotes cell proliferation and invasion via upregulation of the RhoA/Rac2 signaling. Int J Oncol 48: 1590-1598, 2016.

14. Wang X, Sun W, Shen W, Xia M, Chen C, Xiang D, Ning B, Cui X, Li H, Li X, et al: Long non-coding RNA DILC represses self-renewal of liver cancer stem cells via inhibiting autocrine IL-6/STAT3 axis. J Hepatol 64: 1283-1294, 2016.

15. Zhou J, Li M, Yu W, Li W, Wang J, Xiang X, Li G, Pan X and Lei D: AB209630, a long non-coding RNA decreased expression in hypopharyngeal squamous cell carcinoma, influences proliferation, invasion, metastasis, and survival. Oncotarget 7: 14628-14638, 2016.

16. He R, Yang L, Lin X, Chen X, Lin X, Wei F, Liang X, Luo Y, Wu Y, Gan T, et al: MiR-30a-5p suppresses cell growth and enhances apoptosis of hepatocellular carcinoma cells via targeting AEG-1. Int J Clin Exp Pathol 8: 15632-15641, 2015.

17. Eggert T, McGlynn KA, Duffy A, Manns MP, Greten TF and Altekruse SF: Epidemiology of fibrolamellar hepatocellular carcinoma in the USA, 2000-10. Gut 62: 1667-1668, 2013.

18. El-Serag HB: Epidemiology of hepatocellular carcinoma in USA. Hepatol Res 37 (Suppl 2): S88-S94, 2007.

19. European Association For The Study Of The Liver; European Organisation For Research And Treatment Of Cancer: ASL-EORTC clinical practice guidelines: Management of hepatocellular carcinoma. J Hepatol 56: 908-943, 2012.

20. Abdo AA, Hassanain M, AlJumah A, Al Olayan A, Sanai FM, Alsuhaibani HA, Abdulkareem H, Abdallah K, AlMuaikeel M, Al Saghier M, et al: Saudi guidelines for the diagnosis and management of hepatocellular carcinoma: Technical review and practice guidelines. Ann Saudi Med 32: 174-199, 2012.

21. European Association for Study of Liver; European Organisation for Research and Treatment of Cancer: EASL-EORTC clinical practice guidelines: Management of hepatocellular carcinoma. Eur J Cancer 48: 599-641, 2012.

22. Chatterjee R and Mitra A: An overview of effective therapies and recent advances in biomarkers for chronic liver diseases and associated liver cancer. Int Immunopharmacol 24: 335-345, 2015.

23. Ma X, Hui H, Jin Y, Dong D, Liang X, Yang X, Tan K, Dai Z, Cheng $Z$ and Tian J: Enhanced immunotherapy of SM5-1 in hepatocellular carcinoma by conjugating with gold nanoparticles and its in vivo bioluminescence tomographic evaluation. Biomaterials 87: 46-56, 2016.

24. Jaganathan A, Murugan K, Panneerselvam C, Madhiyazhagan P, Dinesh D, Vadivalagan C, Aziz AT, Chandramohan B, Suresh U, Rajaganesh R, et al: Earthworm-mediated synthesis of silver nanoparticles: A potent tool against hepatocellular carcinoma, Plasmodium falciparum parasites and malaria mosquitoes. Parasitol Int 65: 276-284, 2016.

25. Sanchez Y and Huarte M: Long non-coding RNAs: Challenges for diagnosis and therapies. Nucleic Acid Ther 23: 15-20, 2013.

26. Gupta RA, Shah N, Wang KC, Kim J, Horlings HM, Wong DJ, Tsai MC, Hung T, Argani P, Rinn JL, et al: Long non-coding RNA HOTAIR reprograms chromatin state to promote cancer metastasis. Nature 464: 1071-1076, 2010.

27. Fu X, Ravindranath L, Tran N, Petrovics G and Srivastava S: Regulation of apoptosis by a prostate-specific and prostate cancer-associated noncoding gene, PCGEM1. DNA Cell Biol 25: 135-141, 2006.

28. Zhou JY, Li W, Jin T, Xiang X, Li M, Wang J, Li G, Pan X and Lei D: Gene microarray analysis of IncRNA and mRNA expression profiles in patients with hypopharyngeal squamous cell carcinoma. Int J Clin Exp Med 8: 4862-4882, 2015.

29. Spencer SL and Sorger PK: Measuring and modeling apoptosis in single cells. Cell 144: 926-939, 2011.

30. Du Y, Gong J, Tian X, Yan X, Guo T, Huang M, Zhang B, $\mathrm{Hu} \mathrm{X}$, Liu H, Wang Y, et al: Japonicone A inhibits the growth of non-small cell lung cancer cells via mitochondria-mediated pathways. Tumor Biol 36: 7473-7482, 2015. 